\title{
Childhood Ataxia: Current Understanding and Future Directions
}

\author{
Bernard L. Maria, MD, MBA' \\ 'Department of Pediatrics, Georgia Health Sciences University, Augusta, Georgia
}

Childhood ataxia was chosen as the topic for the 11th Neurobiology of Disease in Children Symposium, which was held on October 26, 2011, in Savannah, Georgia. Program codirectors Dr David Lynch and Dr Susan Perlman prepared an outstanding agenda that included individual presentations by and a panel discussion with highly respected leaders in the field of childhood ataxia.

The symposium covered the clinical features, recent advances in understanding the pathogenesis, and new investigations of characteristic symptoms of Friedreich ataxia. This was followed by a session on controversial topics and unanswered questions. The symposium concluded with a panel discussion consisting of scientific leaders in the field of ataxia. Dr Brent Fogel opened the symposium giving an overview of childhood ataxia, beginning with a general definition of ataxia and moving deeper into specific causes and classifications of childhood ataxia - chiefly cerebellar ataxias. After the introduction, Dr Martin Delatycki's presentation focused on the clinical features of Friedreich ataxia: progressive ataxia of limbs, absent lower limb reflexes, and extensor plantar responses to name a few. The next presentation was given by Dr Mark Payne which discussed heart disease in Friedreich ataxia. To this end, Dr Payne pointed out important clinical issues regarding the heart in patients with Friedreich ataxia and identified important questions and findings for the heart in this disease. Ms Jennifer Farmer concluded the first session of presentations and spoke about further details on the genetics of Friedreich ataxia. In addition, she stressed the importance of clinical research studies and the crucial role of advocacy groups.

The next session of the symposium focused on the progress in understanding the mechanisms behind Friedreich ataxia and the progress of the clinical trials. Dr Massimo Pandolfo described how changes in biological pathways related to Friedreich ataxia can result in mitochondrial dysfunction, production of free radicals, and changes in iron metabolism. These consequences due to low levels of frataxin are highlighted in development of therapies as presented by the other speakers. Dr Joel Gottesfeld explained the development of epigenetic controls. With the use of Friedreich ataxia induced pluripotent stem cells, histone deacetylase inhibitors have been developed to prevent frataxin gene silencing. Other studies were explained by Dr Rob Wilson that targeted the three main consequences of altered levels of frataxin. Dr Wilson emphasized how the therapeutic treatments could target different areas that disrupt iron-sulfur cluster synthesis. Dr Susan Perlman concluded the session with her presentation on clinical trials. The lack of proven disease-modifying therapies for Friedreich ataxia leaves room for growth as the number of clinical trials has risen exponentially since 1997. Registries have created a

Corresponding Author: Dr Bernard L. Maria, Department of Pediatrics, Medical College of Georgia, Georgia Health Sciences University, 1446 Harper Street, BT-1850, Augusta, Georgia 30912-3700; bmaria@georgiahealth.edu.

The authors disclosed receipt of the following financial support for the research and/or authorship of this article: Supported by grants from the National Institute of Neurological Disorders and Stroke (5R13NS040925-15), the National Institutes of Health Office of Rare Disease Research, the Child Neurology Society, and the National Ataxia Foundation. 
network of patients who can unite with the research field by participating in the clinical trials. The presentations given by Drs Pandolfo, Gottesfeld, Wilson, and Perlman provided information about the advancements in the study of Friedreich ataxia and set the foundation for new developments.

New investigations in Friedreich ataxia were discussed in the third session of the symposium. Dr David Lynch gave an overview of clinical measures and how these measures are developed. He emphasized the need for targeted therapies that show validation of measures in order to reach phase 3 trials. The presentation by Dr Lynch directed the audience to think of how specific studies could be structured. The session proceeded to go into depth about diabetes and Friedreich ataxia. Dr Steve Willi spoke about the prevalence of insulin resistance in patients with Friedreich ataxia and how improvements in $\beta$-cell function provided significant change. Another disorder present in patients with Friedreich ataxia discussed was cardiac disease. Dr Subha Raman explained the presentation of cardiac disease in patients and how data can be collected to provide targeted therapeutics to prevent disease progression. Next, Dr Gary Rance presented on speech perception and hearing difficulties associated with patients that have Friedreich ataxia. Dr Grace Yoon shared new dilemmas when diagnosing Friedreich ataxia while focusing on pre-symptomatic genetic testing. Dr Yoon pointed out that more attention needs to be brought to evidence-based studies. After an executive summary of the day's presentations by Dr Gihan Tennekoon, the conference concluded with a panel discussion moderated by Dr Katrina Gwinn that addressed future directions in regards to Friedreich ataxia as well as remaining questions for the day's presenters.

The article written by Ashley et al in this issue gives a detailed review of each presentation as well as the valuable verbatim transcript of the questions and answers after each session. The remainder of the article provides valuable insights into diagnosis, management, and the future directions of childhood ataxia. Furthermore, each presenter recorded a video podcast summarizing their presentation, and the podcast was uploaded to the symposium website. ${ }^{1}$ These video podcasts are outstanding updates in the field of childhood ataxia as well as a vision of the future work needed to move the field forward.

I express my sincere appreciation to the National Institute of Neurobiological Disorders and Stroke, the National Institutes of Health Office of Rare Disease, the Child Neurology Society, and the National Ataxia Foundation for co-sponsoring the conference and the Young Investigator Program, which hosted 21 talented trainees and junior faculty with career interests in pediatric neurosciences and childhood ataxia. I would also like to thank Dr Gihan Tennekoon of the Children's Hospital of Philadelphia for inspiring the young investigators and Dr Story Landis for her continued unwavering support of the Neurobiology of Disease in Children Symposium series.

Lastly, I am grateful for the wisdom and guidance of the symposium co-directors Dr David Lynch and Dr Susan Perlman. Additionally, I am humbled and astounded each year by the remarkable support of the Neurobiology of Disease in Children conferences and by the members of the Child Neurology Society.

I am pleased that Batten disease has been selected as the topic for the $12^{\text {th }}$ Neurobiology of Disease in Children Symposium, which will be held on October 31, 2012, in Huntington Beach, California. Program co-directors Dr Jonathan Mink and Dr David Pearce have worked hard and prepared an exceptional agenda that promises to meet or exceed expectations of loyal child neurology attendees as well as a new group of young investigators orienting to our field. We look forward to seeing you in the gorgeous city of Huntington Beach, California. 


\section{References}

1. [Accessed February 22, 2012] Neurobiology of Disease in Children. http:// www.neurobiologyofdisease.com 